PII: S0147-1767(99)00003-6

\title{
COMMUNICATING INFORMATION IN CONVERSATIONS: A CROSS-CULTURAL COMPARISON
}

\author{
HAN ZAO LI* \\ University of Northern British Columbia, Canada
}

\begin{abstract}
The purpose of this study was to compare the amount of information sent by the speaker, received and retrieved by the listener in inter-and intra-cultural conversations. Three hypotheses were tested: (1) in their conversations, inter-cultural interactants would communicate significantly less information than intra-cultural interactants with other variables held constant, (2) the two inter-cultural conditions would not be different from each other in terms of the amount of information communicated, and the same would be true with the two intra-cultural conditions, and (3) in their conversations, the speakers in inter-cultural conditions would send the same amount of information as speakers in intracultural conditions given that all speakers would pass a test on the materials they were going to present. Participants were 40 Canadian and 40 Chinese university students, and they were randomly assigned to one of the four experimental conditions. Each dyad engaged in two medical conversations, which were videotaped upon the consent of the participants. The nature of the conversations resembled physician-patient face-to-face interactions. Results from written tests immediately following the conversations provided strong support for Hypothesis 1 and 2, and results from viewing the videotapes provided partial support for Hypothesis 3. In relation to the amount of information sent by the speakers, listeners in inter-and intra-cultural conditions retrieved only $50 \%$ and $75 \%$ of the information respectively. Based on the findings, one may argue that inter-cultural communication differs significantly from intra-cultural communication in the amount of information
\end{abstract}

This research is based in part on the author's doctoral thesis presented to the Department of Psychology at the University of Victoria. An earlier version of this article was presented at the 45th Annual Conference of the International Communication Association, Albuquerque, New Mexico, May 25-29, 1995.

*The author would like to thank Dr Ronald Hoppe for helpful comments on the manuscript and for support in completing this study; Dr Janet Bavelas, for crucial guidance in designing and conducting the experiment; Dr Michael Bond for enlightening suggestions on the design of the experiment; Drs Lorne Rosenblood, Bruno Zumbo, Peter MacMillan, Cindy Hardy, and Pat Konkin, and Trudy Johnson, for statistical consulting and helpful comments on the manuscript; and all the participants, for their time and efforts in making this study possible.Finally, the author would like to thank the anonymous reviewers for insightful comments.

*University of Northern British Columbia, Department of Psychology, 3333 University Way, Prince George, B.C. Canada V2N 4Z9, Canada. Tel.: +250-960-6502; Fax: + 250960-5536.; E-mail: lih@unbc.ca 
communicated in situations where the second-language speaker has sufficient language ability to participate in the conversation, thus indicating that language ability alone does not guarantee effective inter-cultural communication. The findings of this study have important implications for inter-cultural communication training and health communication. What is more, they further our understanding of the nature of human communication in that what is said by the speaker is not always received, comprehended, or retrieved correctly by the listener. This is true even in intra-cultural interactions, although the information loss is not as severe as in inter-cultural interactions (25\% versus $50 \%)$. Therefore, one may conclude that one cannot not miscommunicate in a discursive situation. (C) 1999 Elsevier Science Ltd. All rights reserved

KEY WORDS. Inter-cultural communication, miscommunication, health communication.

"If we understand others' languages, but not their cultures, we can make fluent fools of ourselves." - Gudykunst, 1991

\section{INTRODUCTION AND LITERATURE REVIEW}

Pragmatically defined, face-to-face communication has two levels: content and relationship (Watzlawick, Bavelas, \& Jackson, 1967). The former refers to conveying "the content of the message", whereas the latter emphasizes elements constituting the on-going relationship between communicants (Watzlawick et al., 1967:51-52; Clark \& Brennan, 1991).

A review of literature in inter-cultural communication indicates that researchers have mainly explored the "relationship" level of communication, but made the "content" level scarce. For example, scholars have examined cross-cultural communication styles (Hall, 1976; Pierson \& Bond, 1982; Ting-Toomey, 1988), linguistic characteristics of cross-cultural interactants (Giles, 1978; Giles \& Franklyn-Stokes, 1989) intercultural adaptation (Berry, 1980; Collett, 1971; Gao \& Gudykunst, 1990; Kim, 1978, 1989), similarities and differences in values, norms, attitudes, and perceptions (Bond, 1991; Gudykunst, Yoon, \& Nishida, 1987; Iwao \& Triandis, 1993; Triandis, Leung, Villareal, \& Clack, 1985), relationship rules (Argyle, Henderson, Bond, Iizuka, \& Contarello, 1986; Triandis, Bontempo, Villareal, Asia, \& Lucca, 1988), and cross-cultural perceptions and observations of facial expressions and communicative body movements (Eibl-Eibesfeldt, 1972; Ekman, 1973; LaFrance \& 
Mayo, 1978; Matsumoto, 1992; Sogon and Masutani, 1989; TingToomey, 1991).

One may argue, however, that an important goal of human face-toface communication is to convey information regardless of the "relationship" between communicants (Weaver, 1966; Wiener, 1966). It is the intention of the present research to study inter-cultural informationcommunication via intra- and inter-cultural comparisons in face-to-face interactions.

First of all, let us define "inter-cultural", "intra-cultural", and "information-communication". According to Hofstede (1980) and Triandis et al. $(1985,1988)$, cultures could be categorized into two types: individualistic and collectivistic. They asserted that North Americans and Europeans are particularly high on ratings of individualism, whereas Asians and Latin Americans are high on collectivism. In terms of communication styles, Hall (1976) differentiated cultures into low and highcontext communication. In low-context communication, interlocutors tend to use a more direct communication style, whereas in high-context communication, interlocutors tend to use a more indirect communication style (Hall, 1976). Gudykunst and Ting-Toomey (1988:44) contended that "the dimensions of low-high-context communication and individualism-collectivism are isomorphic" and "that low- and highcontext communication are the predominant forms of communication in individualistic and collectivistic cultures, respectively". Based on this contention, interactions between collectivistic and individualistic cultures are defined as inter-cultural communication whereas interactions within either of the two cultures are defined as intra-cultural communication. In the present study, intra-cultural communication was defined as either two Canadians, whose first language was English, talking to each other in English, or two Chinese, whose first language was Mandarin Chinese, talking to each other in Mandarin Chinese. Intercultural communication was defined as a Canadian talking to a Chinese or vice versa in English. Since the first language of the Chinese subject was Mandarin Chinese, the Chinese subject would be functioning in his/ her second language.

\section{Information-communication}

Although the information-communication process may be complex, two components are essential: a source which sends the message, and a receiver, that receives the message (Weaver, 1966; Wiener, 1966). To send a message is a communicative act by the source, to receive a message is a communicative act by the receiver, and to communicate the message refers to the whole process which includes acts by the source and the receiver (Weaver, 1966; Wiener, 1966). The more successful the 
information-communication, the smaller the difference between the amount of information sent from the source and information received by the receiver. To study information-communication in face-to-face interactions, the experiment must be designed to allow a comparison of the amount of information sent by the speaker, and received and retrieved by the listener. If we assume that inter-cultural interaction is less successful than intra-cultural interaction, we are predicting that more information is lost for inter-cultural communicants than for intracultural communicants in the information-communication process.

Information-communication in face-to-face interactions was studied by Gardner, Simard and Taylor in the early 1970s (Simard \& Taylor, 1973; Taylor \& Gardner, 1970a,b; Taylor \& Simard, 1975). In three separate experiments, these researchers examined the effectiveness of interethnic communications in comparison to intra-ethnic communication (note that inter-ethnic communication does not necessarily mean intercultural communication). They found that French Canadian/English Canadian communication was as effective as French/French Canadian or English/English Canadian communication. They also found that Chinese Filipino/Tagalog Filipino interaction was as effective as Chinese/Chinese and Tagalog/Tagalog communication.

According to Hofstede (1980) and Triandis et al.'s (1985) categories of culture, Taylor, Gardner and Simard studied inter- and intra-ethnic communication, not inter- and intra-cultural communication. Although French Canadians and English Canadians did not share the same language, they belonged to a similar culture-western culture or individualistic culture. The same was true with Chinese Filipino and Tagalog Filipino. They did not share the same language, but they both belonged to a collectivistic culture. In both cases, inter-ethnic communication was as effective as intra-ethnic communication.

Now the question becomes: what if the interactants do not belong to a similar culture, and one participant of the conversation had to use a second language? Are they capable of communicating effectively given that the second-language user had sufficient language ability to participate in the conversation? Under this circumstance, is inter-cultural communication as effective as intra-cultural communication? If not, what contributes to the miscommunication, language or culture?

Gudykunst (1991:2) pointed out: "if we understand each others' languages, but not their cultures, we can make fluent fools of ourselves". Several researchers have studied natural conversations between interlocutors of different cultures, where the second language speakers had enough language knowledge, but not enough cultural background information, to coordinate the conversation, and their findings have provided strong support for Gudykunst's contention. 
For example, Erickson (1975) videotaped counselling interviews where junior college students were advised on course selections and career choices. He demonstrated that the conversation proceeded more smoothly if the counsellor and student came from similar cultural background (e.g., two Italians). Conversely, the communication was not successful (as measured by the amount of special help the student received from the counsellor) when the counsellor and student were from different cultural backgrounds (e.g., Italian and Polish). The reasons for the poor communication between inter-cultural dyads was attributable to differences in communication styles such as a lack of rhythmic coordination.

Gumperz (1978) analyzed episodes of conversations between bilingual Indian and Pakistani immigrants functioning in Britain, and found that they often had problems communicating with native British. "Since Indians and Pakistanis are not native speakers of English, one might assume that their communication problems are simply due to their lack of knowledge of the language (1978:17)", but that does not seem to be the case. Among Indians and Pakistani immigrants in England, those who knew English well had as many disputes (with native British) attributable to communication failures, as those who had little English. Gumperz (1978:18) then asserted:

Clearly the problem is not simply knowledge of the language. Nor is the question simply one of foreign accent . . Greek and Cypriot immigrants often have accents which, measured in purely linguistic terms, are even more deviant than those of Indians and Pakistanis, but yet they have less difficulty communicating.

Analyzing job interviews of intra- and inter-cultural dyads, Gumperz $(1992: 320,324)$ noticed that, when interviewed by native English speakers, responses by Asian bilinguals "are patterned with culturally based differences in communication style, differences which significantly affect the quality of the interaction". In contrast, talks between two native English speakers flowed smoothly characterizing "synchronized exchange".

Tannen (1981) studied Greek-American conversations and discovered that miscommunication was a result of directness-indirectness differences. Further, listener interpretation of the speaker was influenced by the listener's "sociocultural knowledge" (Tannen, 1981:234). Among intra-cultural interlocutors, there was a "sharing of conversational strategies that creates the feeling of satisfaction which accompanies and follows successful conversation ... Conversely, a lack of congruity in conversational strategies" in inter-cultural interlocutors lead to miscommunication. 
Talking from twenty years of research on inter-cultural communication in North America and Asia, Scollon and Scollon (1995) concluded that "most miscommunication doesn't arise through mispronunciations or through poor uses of grammar ..., rather lies in differences in patterns of discourse" (p. xii).

Gass and Varonis $(1984,1985,1991)$ collected episodes of phone and face-to-face conversations between intercultural interlocutors (one was a native speaker of the language used in the conversation, and the other was a non-native speaker), they found that "miscommunication" was "abound", even when the second language speaker had a certain level of language fluency.

To sum up, there seemed to be a consensus that fluency in language per se did not guarantee accuracy in inter-cultural communication (Hammerly, 1991). Major barriers to inter-cultural communication may not lie in a lack of linguistic skills per se, rather, in differences in cultural backgrounds and their consequences on communication.

Based on the above arguments, the author proposed three hypotheses for the present study. Hypothesis 1 predicted that in their conversations, inter-cultural interactants would communicate significantly less information than intra-cultural interactants, with other variables held constant. By other variables being constant, it meant that the nature and amount of information to be communicated were the same for interand intra-cultural dyads, and the second-language user had sufficient language ability to be a participant (see Method section for more details).

Hypothesis 2 predicted that the two inter-cultural conditions would not be different from each other in terms of the amount of information communicated, and the same would be true with the two intra-cultural conditions. The rationale for the assumption was that there would not be a significant difference in terms of the amount of information communicated between the two intra-cultural conditions since, in each condition, subjects were functioning in their first language, and communicating with a partner from their own culture. It follows, then, if subjects from two cultures (Canadian vs Chinese) interacted, and one subject (the Chinese) functioned in his/her second language and in a foreign culture, whether he/she were the speaker or the listener, the amount of information lost in the communication process would be similar. Therefore, there would not be a statistically significant difference in terms of the amount of information communicated between the two inter-cultural conditions.

Hypothesis 3 predicted that in their conversations, the speakers in inter-cultural conditions would send the same amount of information as speakers in intra-cultural conditions given that all speakers would pass a test on the materials they were going to present. The assumption for 
this hypothesis was that the second-language speakers would not have language difficulties in presenting the information (see details about this assumption in Method section). Therefore, there should not be a significant difference in the amount of information sent, given that the second language speakers had memorized the information beforehand, and there would be a significant difference in the amount of information received and retrieved by the listeners. The rationale was that barriers other than language would prevent the inter-cultural dyads from getting the information through.

The goal of the present research was to ascertain the amount of information lost in inter-cultural conversations by comparing the amount of information sent by the speaker and retrieved by the listener. To make the assessment of information-communication meaningful, two intracultural conditions (Canadian/Canadian and Chinese/Chinese) were used as comparisons to the two inter-cultural conditions (Canadian/ Chinese and Chinese/Canadian).

Subjects chosen for the present study were 40 Canadians whose first language was English and 40 Chinese whose first language was Mandarin Chinese and grew up in China. The study was conducted in a laboratory setting with assigned topics. Each dyad engaged in two conversations; one was an analogue of taking a personal case history, and the other required communicating instructions about the use of a common medicine. The nature of the conversations resembled physician-patient face-to-face interactions. The rationale for studying medical interviews rather than other types of communication was that among other difficulties immigrants faced, the inability to communicate with health professionals seemed prominent (Lai \& Yue, 1990; Li, 1992; Stephenson, 1991; Woon, 1986). According to findings of a recent health survey in a randomly selected sample of 224 Chinese immigrants in the province of British Columbia, Canada, miscommunication with health professionals of the mainstream culture was identified as a major health problem which had an impact on every other aspect of their life in Canada (Li, 1992). This was true even with the $76.6 \%$ who rated their English as "fluent" or "fair" (Li, 1992). Similar findings were reported among Vietnamese immigrants in Victoria, British Columbia, Canada, that most of their problems "were rooted in the failure to communicate accurately and meaningfully" (Stephenson, 1991:6).

The rationale for choosing simulated physician-patient conversation instead of real physician-patient interaction was that in the latter, it would be impossible to use exactly the same conversation topic for all dyads, thus making measurement of information-communication difficult. 


\section{METHOD}

\section{Subjects}

Eighty-four subjects, 44 males and 40 females, volunteered to participate in this study. The subjects formed 42 same-gender dyads, two of which were dropped from data analysis because they did not follow the instructions, leaving a total of 40 dyads. ${ }^{1}$ Subjects were either thirdyear, fourth-year, or graduate students enrolled at the University of Victoria. The majority of the subjects were in their twenties and early thirties with an average age of 29.11. The mean age for the Chinese subjects was 30.0 and that for the Canadian subjects was 28.2. ${ }^{2}$ Subjects were solicited in classrooms, the university cafeterias and graduate students' offices in various departments at the University of Victoria. In their first encounter with the experimenter, subjects were informed of the nature of the study and that they were going to be videotaped should they agree to participate. Upon arrival at the Human Interaction Laboratory, subjects were told again that their conversations would be videotaped and that they could view their own tape afterwards if they wished. Upon finishing the experiment, written consent was obtained from each subject regarding the way(s) in which the videotape made during the experiment might be used. Among the six options, one was "please erase the tape" ( $\mathrm{Li}, 1994)$.

Of the 80 subjects, 40 were Chinese whose first language was Mandarin Chinese, and 40 were Canadians whose first language was English. All Chinese subjects grew up in Mainland China and were studying at the University of Victoria, Canada at the time of the experiment. To ensure that the second language speakers have sufficient language ability to participate in the conversations, the following measures were taken into consideration. First, all subjects were given time to learn the testing materials and took a multiple-choice test of the testing materials before they started their interactions (see Procedures section for details).

Second, speakers could have their information sheet available during the conversations, but were not to read from it word for word or show it to the listener (Procedure).

Third, all conversations were video-taped, speakers' ability to send the information verbally was measured by the unit of information elicited correctly (Hypothesis 3).

${ }^{1}$ One dyad started to laugh in the middle of the first dialogue and the laughing continued for $60 \mathrm{~s}$. The other dyad did not finish the second dialogue because the speaker was not feeling well.

${ }^{2}$ The means were not significantly different from each other $(t(78)=1.90, P>0.05)$. 
Fourth, upon finishing the conversations, all inter-cultural dyads filled out a questionnaire to inquire if they experienced any communication difficulties in the interactions. On a scale from 0 "not at all" to 6 "very difficult", the mean score for the Canadian subjects was 1.32 (S.D. $=1.29)$ and the mean score for the Chinese subjects was 1.42 $($ S.D. $=1.50)$. The mean scores for the Canadians and Chinese were not significantly different $(t(38)=.23, P>0.05)$. It appeared that inter-cultural interactants felt that they experienced very few difficulties in their communication. In addition, Canadian subjects were asked to rate their Chinese partners' English fluency, and Chinese subjects rated their own English fluency. In general, Canadians rated their Chinese partners fairly high on English fluency $(M=4.0$, S.D. $=0.66$, on a scale from 0 "not fluent" to 6 "very fluent"), and Chinese rated their own English fluency also fairly high $(M=3.32$, S.D. $=1.75)$.

Lastly, TOEFL scores were used as a reference for reading and listening comprehension. All Chinese subjects had a TOEFL score of 550 or above.

At the time of the experiment, the Chinese subjects had resided in Canada for an average of 27 months, with a range of 0.5 to 60 months. The experiment was conducted from September to November, 1993.

\section{Experimental Design}

A between-subjects design with four conditions was used: Canadian/ Canadian, Chinese/Chinese, Canadian physician/Chinese patient, and Chinese physician/Canadian patient. Subjects were randomly assigned to one of the three conditions based on their available time. A Canadian participant was randomly assigned to one of the following three conditions: Canadian/Canadian; Canadian physician/Chinese patient, and Chinese physician/Canadian patient. In the same way, a Chinese participant was assigned to one of the three conditions: Chinese/Chinese, Canadian physician/Chinese patient, and Chinese physician/Canadian patient. In each condition, one person played the role of a physician and the other the role of a patient. In the intra-cultural conditions (i.e., the Canadian/Canadian and Chinese/Chinese), the roles of physician and patient were randomly assigned. In the inter-cultural conditions (i.e., Canadian physician/Chinese patient and Chinese physician/Canadian patient), only half of the roles of physician and patient could be randomly assigned. For the first two inter-cultural dyads (Canadian physician/Chinese patient and Chinese physician/ Canadian patient), the roles of physician and patient were randomly assigned. For the last two inter-cultural dyads, the roles of physician and patient were fixed. The experiment was done in blocks, every eight 
dyads forming a block. In every block, two dyads were required in each of the four conditions.

In all 40 dyads, males were paired with males and females were paired with females. In each of the four conditions, males and females were evenly distributed so that 5 male and 5 female dyads were in each condition.

\section{Testing Materials and Translation}

The two dialogues for the experiment were analogues of taking a personal case history and of giving instructions about the use of a common medicine. In developing the testing materials, repeated pretests were conducted with Canadian and Chinese students. After many modifications based on the experience and suggestions of these pilot subjects, the final version came into being ${ }^{3}(\mathrm{Li}, 1994)$.

All materials had English and Chinese versions. The CanadianCanadian and Canadian-Chinese dyads used English, and the ChineseChinese dyads used Chinese. The method used for translation was a combination of back translation and the bilingual technique as recommended by Brislin (1980). The first version was written in Chinese because the original story was told by a Chinese immigrant. Two bilingual graduate students then translated the Chinese story into English. In the course of translation, a combination of literal and free translation was applied (Brislin, 1980). Due to the differences in the two languages and cultures, some difficulty was encountered in finding English equivalents for some Chinese sentences. For example, the usual way a Chinese physician greets a patient when he/she comes into the office is: "So, which part of your body bothers you today?" By being specific ("which part of your body"), the physician conveys the message that he/she is sincerely concerned with the condition of the patient. Physicians in Canada, however, usually don't ask such a question of their patients. After consulting a psycholinguist and a linguist, it was decided to compromise between a literal and a free translation. The final version became "So, what seems to be the problem?" The English version was then translated back into Chinese. In the open-ended tests of both dialogues, the Chinese patient in the inter-cultural interaction could choose to write the test in either Chinese or English.

\section{Procedures}

There were two phases, each comprising of three steps, that were followed in preparation for performing Dialogues 1 and 2. Phase one con-

\footnotetext{
${ }^{3}$ All testing materials and scoring standards are available from the author upon request.
} 
sisted of three steps: (a) one subject adopted the case history of a 65year-old patient who was suffering from chronic arthritis and recent chest pains (based on the true story of a Chinese immigrant). The subject was given time to learn the details of the case and then took a multiple-choice test to ensure that he or she had adequately mastered the role to be played; (b) the two subjects interacted freely, with one playing the role of a family physician whose task was to learn the medically relevant facts from the patient; (c) the subject with the role of physician took an open-ended test to measure how much information about the case history was successfully communicated.

In the second phase, the subject playing the role of physician was first given information on the use of codeine (taken from Compendium of pharmaceuticals and specialties, 1982), which was appropriate for the case history just presented. After studying the information, the subject took a multiple-choice test to ensure that he or she had adequately mastered the role to be played. Second, the two subjects interacted again. This time, the physician informed the patient about the use of codeine. Lastly, the subject playing the role of the patient took an open-ended test, which measured how much information about the medication was successfully communicated.

In performing the dialogues, the following guidelines were followed. (a) neither physician nor patient was given specific instructions regarding how to pass the information. That is, the physician was not given a set format for asking questions or explaining codeine, and the patient was not told how to tell his or her case history or what to ask about the medication; (b) to minimize purely memory problems, both patient and physician could have their information sheet available during the interaction but were not to read from it or show it to the other; (c) subjects were instructed to convey the information in a natural, "talking" manner ( $\mathrm{Li}, 1994)$.

\section{Scoring}

First, an answer key was developed for the two open-ended tests $(\mathrm{Li}$, 1994). The first test (for Dialogue 1) consisted of 10 open-ended questions. Questions 1-5, 7 and 8 were each assigned 4 points; Question 6 was assigned 12 points, and Questions 9 and 10 were each assigned 8 points. The total points for Test 1 (for Dialogue 1) was 56 . The second test (for Dialogue 2) was made up of 7 open-ended questions. Questions 1, 5, 6 and 7 were each assigned 4 points; Question 2 was assigned 28 points, Question 3 was assigned 8 points, and Question 4 was assigned 16 points. The total points for Test 2 (for Dialogue 2) was 68 .

The points for each question were allocated on the basis of the number of information units ( $\mathrm{Li}, 1994)$. The smallest string of words with 
meaning was the unit of analysis (Bales, 1950; Hall et al., 1994; Roter, 1977; Roter \& Hall, 1992). Each unit of information was worth 4 points. Some questions required answers containing one unit of information while others contained several units of information. Thus some questions were valued at 4 points, while others were as high as 28 points. For example, the answer to Question 1 in Test 1 "why did the patient come to see you" only contained one unit of information: chest pains; whereas, answers to Question 2 in Test 2 "what are the possible side effects after you take codeine" contained 7 units of information: drowsiness, nausea, vomiting, constipation, an increase in heart rate, agitation, and respiratory problems. Thus Question 2 in Test 2 was worth 28 points.

For each question, one or several correct answers were provided in the "Answer Key" ( $\mathrm{Li}, 1994)$. If the answer was essentially identical to the key, it was scored as 4 . If the answer was very close to the correct answer, it was scored as 3 . If the answer was related to the correct answer (e.g., describing but not naming), it was scored as 2 . If the answer was remotely related to the correct answer in that the meaning could be inferred, it was scored as 1 . Blank or wrong answers were scored as 0 . A scale of $0-4$ allowed the scorer to assign appropriate scores to the range of answers given.

For example, Question 7 in Test 1 was: what was the main reason the patient went swimming? If the answer was "the reason the patient went swimming was to exercise his/her legs; or to exercise his/her legs", it was scored as 4. If the answer was essentially "swimming is good for his/her legs", it was scored as 3. If the answer was essentially "to do exercise because the patient has difficulties walking", it was scored as 2 . If the answer was essentially "for exercise or to stay fit", it was scored as 1 .

\section{Inter-scorer Reliability}

Before scoring the data, scorers received a training session with the following instructions: (1) Read criteria at least twice; (2) watch the tape and digest scoring criteria; (3) score for the first time by watching the tape and listening to the dialogue; (4) score for the second time without watching the tape so you can just concentrate on the conversations.

During the training session, scorers summarized the following possible difficulties to be aware of: (1) rush to the task without understanding the scoring criteria thoroughly; (2) when scoring, we are tempted to be carried away by the story and the performance (e.g., humorous remarks and expressive facial expressions); (3) task oriented (e.g., unwillingness to go back and listen for another time when we were not sure). 


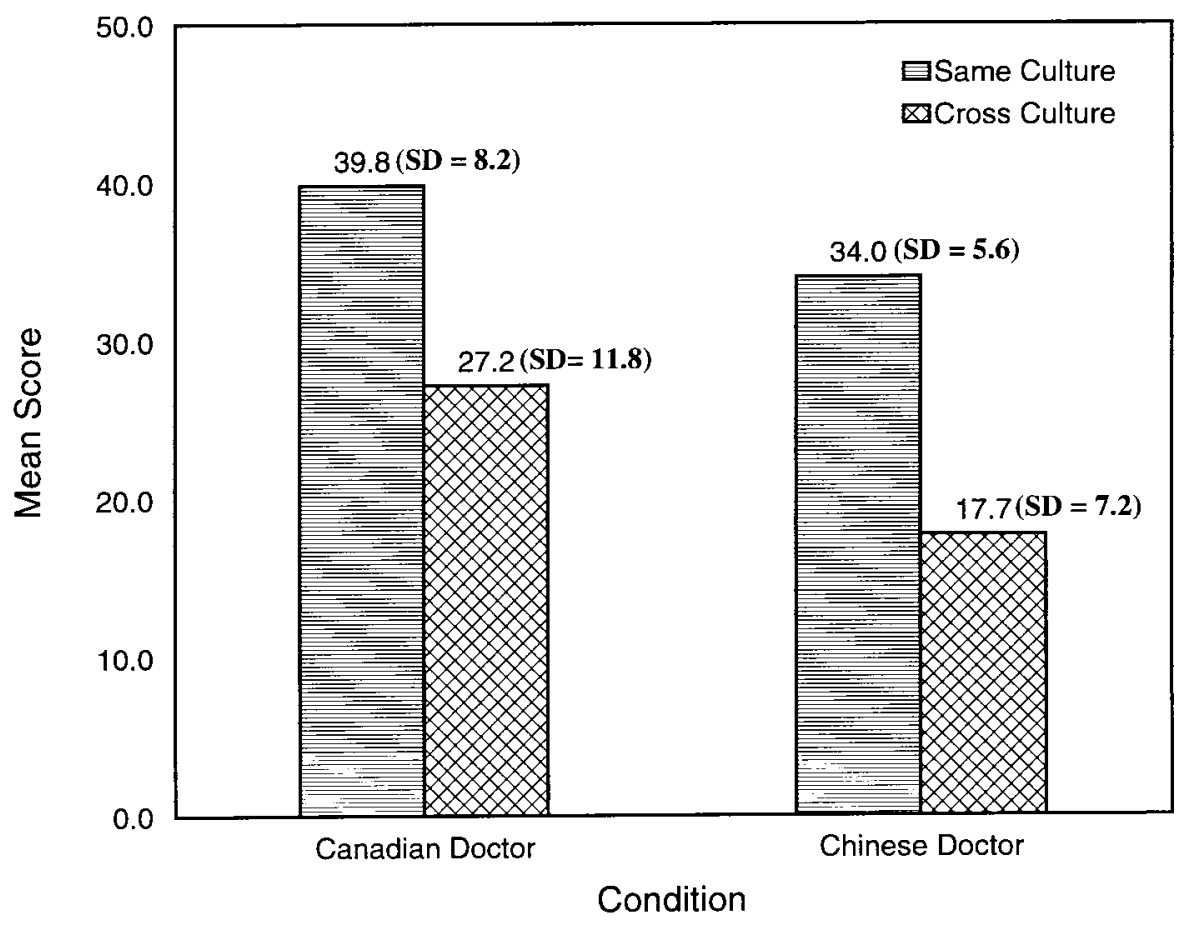

FIGURE 1. Open-ended Test Scores by Condition (Dialogue 1).

Following the training session, three persons independently scored the open-ended tests and the inter-scorer reliability was between 0.98 and 0.99 . The main reason for the high reliability was that almost all subjects either recalled the information or did not. Scorers rarely needed to make a decision on the number of points given to a particular answer. Another reason was that the scoring system was appropriate to the tests. Originally, this scoring system was developed to score the pilot data. It proved to be a sensitive measure for the answers to the tests given by the pilot subjects. Therefore, no adjustments were needed when applied to the present data.

Several factors minimized potential scorer bias. First, facts were being scored. That is, the unit of information was either present or not. Second, the scorers did not have to make a decision based on personal impression or perception of the subjects, which could easily have lead to scorer bias. Finally, the two scorers who had no knowledge regarding 


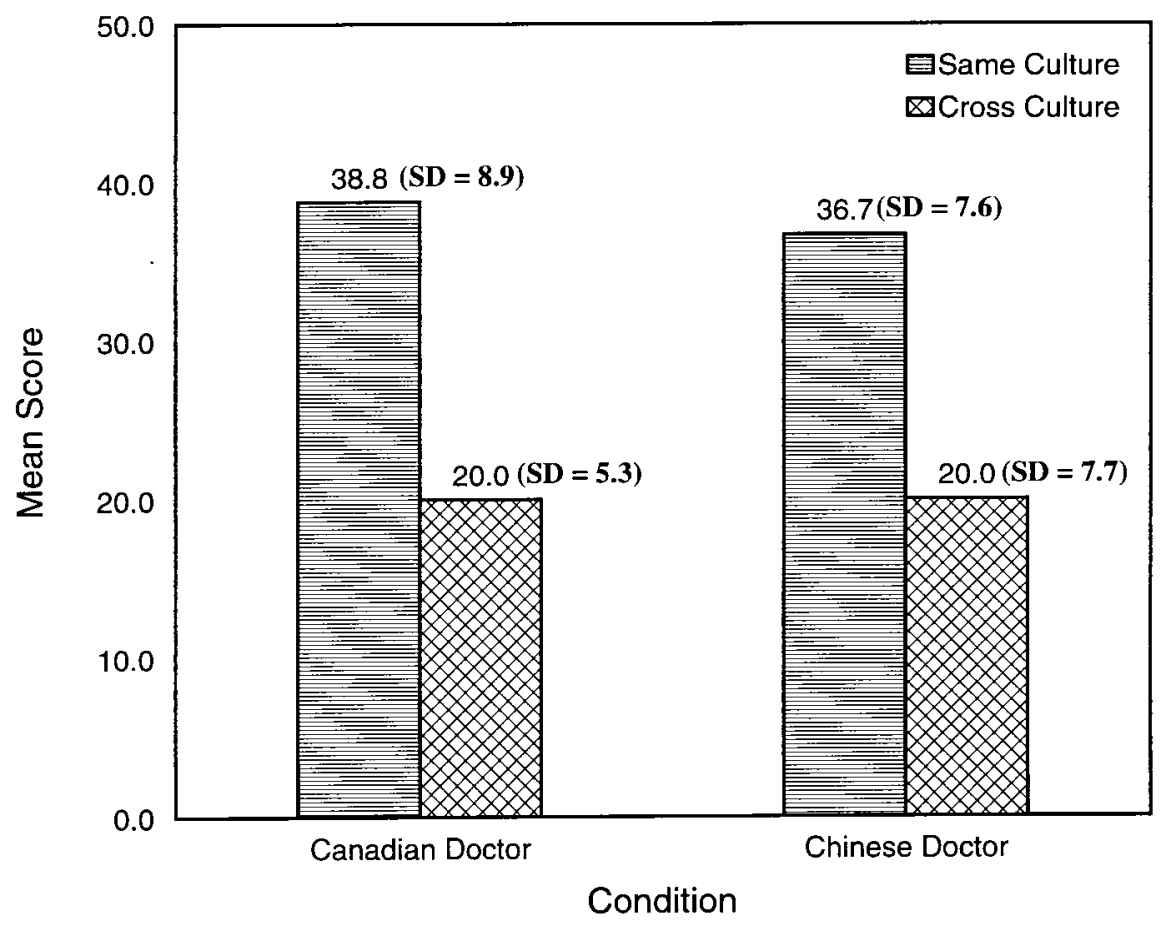

FIGURE 2. Open-ended Test Scores by Condition (Dialogue 2).

the nature of the hypotheses achieved as high a reliability score between themselves as with the primary researcher.

\section{RESULTS}

\section{Testing Hypothesis 1}

To test Hypothesis 1 which predicted that, in their conversations, inter-cultural interactants would communicate significantly less information than intra-cultural interactants, the mean scores from the openended tests for Dialogues 1 and 2 (presented in Figures 1 and 2) were compared using $t$-tests. In all four conditions, $n=10$, where $n$ represents the number of dyads. All dyads were same-gender; males and females were evenly distributed in all conditions. For Dialogue 1, the lowest score was 4 and the highest was 49, while for Dialogue 2, the lowest score was 4 and the highest 48. As shown in Figures 1 and 2, dyads in 
the two intra-cultural conditions achieved higher scores for both dialogues than dyads in the two inter-cultural conditions.

For the open-ended test scores, results of $t$-tests indicated that significant differences existed between the means of intra- (Canadian/ Canadian and Chinese/Chinese) and inter-cultural (Canadian physician/ Chinese patient and Chinese physician/Canadian patient) dyads for both Dialogue $1(t(38)=4.97, P<0.0001)$, and Dialogue $2(t$ $(38)=7.65, P<0.0001)$. Levene's test for equality of variance showed no significant difference between intra- and inter-cultural dyads for Dialogue $1(F=2.06, P>0.05)$, nor for Dialogue $2(F=1.61, P>0.05)$. Thus, the data provided strong support for Hypothesis 1 .

\section{Testing Hypothesis 2}

While Hypothesis 1 tested that subjects in the two inter-cultural conditions would communicate significantly less information than subjects in the two intra-cultural conditions, Hypothesis 2 predicted that the two inter-cultural conditions would not be different from each other in terms of the amount of information communicated, and the same would be true with the two intra-cultural conditions. To test Hypothesis 2, planned comparisons were made, at an $\alpha$ level of 0.05 , to contrast mean scores between the two intra-cultural conditions (Canadian/Canadian and Chinese/Chinese), as well as the two inter-cultural conditions (Canadian physician/Chinese patient and Chinese physician/Canadian patient).

The results showed that the two intra-cultural conditions did not differ significantly from each other for Dialogue 1 and Dialogue 2 ( $F(2$, $\left.35)=1.2, P>0.05 ; \eta^{2}=0.10\right)$, and the two inter-cultural conditions did not differ significantly from each other for Dialogue 1 and Dialogue 2 $\left(F(2,35)=3.1, P>0.05 ; \eta^{2}=0.15\right)$. Thus Hypothesis 2 was supported by the data.

\section{Testing Hypothesis 3}

Using the same scoring standards as used for the open-ended tests, the videotaped interactions revealed that speakers in each of the four conditions sent similar amount of information for Dialogue 1 (see Table 1). In Dialogue 2, speakers in the Canadian/Canadian, Chinese/Chinese, and Canadian physician/Chinese patient conditions also sent similar amount of information (see Table 1). However, the mean score for the Chinese physician/Canadian patient condition was some 10 points lower than the other three conditions.

Results of Univariate $F$-tests indicated no significant difference between the mean scores of intra- (Canadian/Canadian and Chinese/ 
Chinese) and inter-cultural (Canadian physician/Chinese patient and Chinese physician/Canadian patient) dyads for Dialogue 1 ( $F(1$, $38)=2.02, P>0.05)$, but a slight difference was found in Dialogue $2(F$ $(1,38)=7.12, P=0.01)$. Thus, Hypothesis 3 was supported by the data for Dialogue 1.

When testing a hypothesis of "no difference" between the experimental conditions, $\beta$, the probability of a Type II error, and Power $(1-\beta)$ should be major concerns (Cohen, 1977). Cohen (1977) pointed out that a more powerful experiment ( power $=0.80$ or above) has a better chance of rejecting a false null hypothesis than does a less powerful experiment. As power is influenced by several parameters including sample size, a relatively small power (less than 0.80 ) would be expected in the present study due to the size of the sample ( $N=20$ dyads in each group). In situations such as this, Cohen (1977) recommended the consideration of effect size $(\gamma)$, which is a measure of the degree to which the two sample means differ in terms of standard deviation of the parent population.

When a Multivariate Analysis of Variance (SPSS V6.1) of the scores of the speakers in the inter- and intra-cultural conditions was carried out, power was calculated to be $0.75, \beta=0.25$, and $\gamma=0.20$ at an $\alpha$ level of 0.05 . A power of 0.75 indicated a $75 \%$ chance of rejecting Hypothesis 3 , that there was no statistically significant difference between the mean scores of inter- and intra-cultural speakers, when in fact a difference existed. In other words, a $\beta$ of 0.25 indicated a $25 \%$ chance of accepting Hypothesis 3 that there was no statistically significant difference between the mean scores of inter- and intra-cultural speakers, when in fact a difference existed. However, all is not lost. An examination of the effect size provided another indicator of support for Hypothesis 3. The

TABLE 1

Mean Scores from Video-tape by Condition ${ }^{a}$

\begin{tabular}{|c|c|c|c|c|c|c|c|}
\hline \multirow[b]{2}{*}{ Condition } & \multirow[b]{2}{*}{$n$} & \multicolumn{2}{|c|}{ Dialogue 1} & \multicolumn{2}{|c|}{ Dialogue 2} & \multicolumn{2}{|c|}{ Total } \\
\hline & & $M$ & SD & $M$ & SD & $M$ & SD \\
\hline $\mathrm{Ca} / \mathrm{Ca}$ & 10 & 50.4 & 4.8 & 52.0 & 12.9 & 51.2 & 6.2 \\
\hline $\mathrm{Ch} / \mathrm{Ch}$ & 10 & 45.2 & 10.1 & 52.8 & 10.3 & 49.0 & 8.0 \\
\hline $\mathrm{CaDr}$ & 10 & 43.6 & 10.7 & 50.8 & 11.3 & 47.2 & 6.0 \\
\hline $\mathrm{ChDr}$ & 10 & 43.6 & 10.7 & 33.2 & 8.2 & 38.4 & 7.4 \\
\hline
\end{tabular}

${ }^{a} n$ represents the number of dyads. All dyads were same-gender; males and females were evenly distributed in all conditions. 
effect size of 0.20 indicated that the distance between the two sample means was only 0.20 standard deviations, which is considered small (Cohen, 1977), therefore, lending a reasonable level of support for Hypothesis 3.

With respect to the amount of information received as well as retrieved by the listener in comparison to the amount of information sent by the speaker, the ratio was $21.2 / 42.8(50 \%)$ for inter-cultural dyads and $37.3 / 50.1(75 \%)$ for intra-cultural dyads. This difference was statistically significant $\left(\chi^{2}(1, N=40)=6.14, P<0.01\right)$.

\section{DISCUSSION}

The data have provided strong support for Hypothesis 1 that intercultural interlocutors communicated significantly less information than intra-cultural interlocutors. Hypothesis 2 was also supported by the data that the two intra-cultural conditions (Canadian/Canadian and Chinese/Chinese) did not differ from each other in term of the amount of information communicated, and the same was true for the two intercultural conditions. Hypothesis 3 was supported by task 1 that speakers in intra- and inter-cultural conditions sent similar amount of information.

The support for Hypothesis 1 has documented the notion that intercultural communication differs significantly from intra-cultural communication in the amount of information communicated in situations where the second-language speaker has sufficient language ability to participate in the conversation. This finding has an important implication for inter-cultural communication training. To achieve effective inter-cultural communication, we need, first of all, to develop an awareness. We need to be aware that inter- and intra-cultural communications differ greatly in the amount of information communicated even in situations where the second language speaker has sufficient language ability to participate in the conversation. Without this awareness, inter-cultural communicants are highly unlikely to take any measure to facilitate communication in the conversation process.

The confirmation for Hypothesis 2 evidenced that when interlocutors share the same native language and culture, their communication is more efficient than inter-cultural dyads. Gumperz and Tannen (1979:323) observed that communicants "feel more comfortable with those who share one's communicative systems". Simard and Taylor (1973) and Taylor and Simard (1975) reported that interlocutors who didn't share the same language (e.g., French/English Canadians) but came from similar culture (Western culture) had no problem communicating when the second language speaker had language fluency. The critical issue, according to Taylor and Simard $(1973,1975)$, is cultural 
knowledge, which can be a facilitator or inhibitor of inter-ethnic/cultural communication.

The indication of Hypothesis 3 was that in inter-cultural communication, problems can occur even when the speaker has no problem eliciting the information. In other words, the second-language speakers sent as much information as the first language speakers. Nevertheless, listeners in inter-cultural conditions failed to receive and retrieve as much information as listeners in intra-cultural conditions. It is evident that barriers other than language prevented the inter-cultural dyads from getting the information through, i.e., more communication difficulties occurred in the information-communication process for the intercultural dyads than for the intra-cultural dyads. Thus, inter-cultural dyads failed to communicate as much information as intra-cultural dyads although the amount of information sent for inter- and intra-cultural conditions was similar. Apparently problems occurred in one or all of the following phases: receiving, comprehending, retaining, and retrieving the information. Tannen (1981) emphasized that sociocultural knowledge influenced interpretation. Gumperz (1978:320) demonstrated that "culturally based differences in communication style" affect understanding and the quality of interaction. Gass and Varonis (1991:122) contended that "the possibility for miscommunication is profound" when communicants don't share "sociocultural rules of discourse".

It may be argued that to interact proficiently, one needs to know not only language but also the hidden and unhidden cultural rules of one's interactant with regard to anything about the conversation. The knowledge of culturally specific rules of discourse (Labov \& Fanshel, 1977) allows one to feel at ease in the conversation process, as culture for people is like water for fish (Linton, 1936). Language ability, on the other hand, permits one to communicate the verbal messages. Proficiency in cultural rules and language ability together enables one to engage in an effective communication activity. Taken separately, neither one is sufficient in a discursive situation.

The results that listeners in intra- and inter-cultural conditions retrieved only 75 and $50 \%$ of the information sent by the speakers respectively have raised a critical question for health communication. What are the possible consequences of poor physician/patient communication in diagnosis, treatment, and utilization of the health care system? A wrong or poor diagnosis would at best lead to repeated outpatient visits, unneeded hospitalization, and unnecessary expense, and at worst unattainable health outcomes or even death (Makoul, Arntson, \& Schofield, 1995; Sharf, 1990). Misunderstanding of medical recommendations would result in poor cooperation on the part of the patient (DiNicola \& DiMatteo, 1984; Dunbar-Jacob, Dwyer, \& Dunning, 1991), frustration on the part of the physician (Gerber, 1987; Gibbs, 
1989; Wyatt, 1991), and poor prognosis (Diaz-Duque, 1989; Wood, 1989). The end result is an ineffective utilization of the health care system (Guendelman \& Witt, 1991-1992; Masur, 1981).

Due to the small sample size and subsequently a large $\beta$ error (0.25), one must be cautious in the interpretation of "no difference" in testing Hypothesis 3.

In summary, the findings of this study have important implications for inter-cultural communication training and health communication. What is more, they further our understanding of the nature of human communication in that what is said by the speaker is not always received, comprehended, or retrieved correctly by the listener. This is true even in intra-cultural interactions, although the information loss is not as severe as in inter-cultural interactions ( $25 \%$ vs $50 \%)$. Therefore, one may conclude that one cannot not miscommunicate (to follow an expression "one cannot not communicate" by Watzlawick et al., 1967:51) in a discursive situation.

\section{REFERENCES}

Argyle, M., Henderson, M., Bond, M., Iizuka, Y., \& Contarello, A. (1986). Cross-cultural variations in relationship rules. International Journal of Psychology, 21, 287-315.

Bales, R. F. (1950). Interaction process analysis. Cambridge, MA: AddisonWesley.

Berry, J. W. (1980). Acculturation as varieties of adaptation. In A. M. Padilla (Ed.), Acculturation: theory, models and some new findings (pp. 9-25). Washington, DC: Westview.

Bond, M. H. (1991). Beyond the Chinese face. Hong Kong: Oxford University Press.

Brislin, R. (1980). Analysis of oral and written materials. In H. C. Triandis \& J. W. Berry (Eds.), Handbook of cross-cultural psychology: methodology (pp. 389-444). Allyn and Bacon Inc.

Canadian Pharmaceuticals Association (1982). Compendium of pharmaceuticals and specialties: The Canadian preference of health professionals. Toronto: Southam Murray.

Clark, H. H., \& Brennan, S. E. (1991). Grounding in communication. In L. B. Resnick, J. M. Levine \& S. D. Teasley (Eds.), Perceptives on socially shared cognition (pp. 127-149). Washington: APA Books.

Cohen, J. (1977). Statistical power analysis for behavioral sciences (Revised). New York: Academic Press.

Collett, P. (1971). Training Englishmen in the non-verbal behaviour of Arabs: an experiment on intercultural communication. International Journal of Psychology, 6, 209-215. 
Diaz-Duque, O. F. (1989). Communication barriers in medical settings: Hispanics in the United States. International Journal of the Sociology of Language, 79, 93-102.

DiNicola, D. D., \& DiMatteo, M. R. (1984). Practitioners, patients and compliance with medical regimens: a social psychological perspective. In A. Baum, S. E. Taylor \& J. E. Singer (Eds.), Handbook of psychology and health: Social psychological aspects of health (vol. IV). Hillsdale, NJ: Erlbaum.

Dunbar-Jacob, J., Dwyer, K., \& Dunning, E. J. (1991). Compliance with antihypertensive regimen: a review of the research in the 1980 s. Annals of Behavioral Medicine, 13, 31-39.

Eibl-Eibesfeldt, I. (1972). Similarities and differences between cultures in expressive movements. In R. A. Hinde (Ed.), Nonverbal communication (pp. 297312). Cambridge: Cambridge University Press.

Ekman, P. (1973). Cross-cultural studies of facial expression. In P. Ekman (Ed.), Darwin and facial expression: a century of research in review (pp. 169222). New York: Academic Press.

Erickson, F. (1975). Gatekeeping and the melting pot: interaction in counselling encounters. Harvard Educational Review, 45, 44-70.

Gao, G., \& Gudykunst, W. B. (1990). Uncertainty, anxiety, and adaptation. International Journal of Intercultural Relations, 14, 301-317.

Gass, S., \& Varonis, E. (1984). The effect of familiarity on the comprehensibility of nonnative speech. Language Learning, 34, 65-89.

Gass, S., \& Varonis, E. (1985). Variations in native speaker speech modification on nonnative speakers. Studies in Second Language Acquisition, 7, 37-58.

Gass, S., \& Varonis, E. (1991). Miscommunication in nonnative speaker discourse. In N. Coupland, H. Giles \& J. Wiemann (Eds.), Miscommunication and problematic talk (pp. 121-145). Newbury Park, CA: Sage.

Giles, H. (1978). Linguistic differentiation between ethnic groups. In H. Tajfel (Ed.), Differentiation between social groups (pp. 361-393). London: Academic Press.

Giles, H., \& Franklyn-Stokes, A. (1989). Communicator characteristics. In M. K. Asante \& W. B. Gudykunst (Eds.), Handbook of international and intercultural communication (pp. 117-144). CA: Sage Publications.

Gerber, L. A. (1987). Experiences of forgiveness in physicians whose medical treatment was not successful. Psychological Reports, 61, 236.

Gibbs, N. (1989). Sick and Tired: Uneasy patients may be surprised to find that their doctors are worried too. Time, July 31, 48-53.

Gudykunst, W. B. (1991). Bridging differences: effective intergroup communication. Newbury Park, CA: Sage.

Gudykunst, W. B., \& Ting-Toomey, S. (1988). In Culture and interpersonal communication (p. 44). Newbury Park CA: Sage.

Gudykunst, W. B., Yoon, Y., \& Nishida, T. (1987). ]The influence of individualism-collectivism on perceptions of communication in ingroup and outgroup relationships. Communication Monographs, 54, 295-306.

Guendelman, S., \& Witt, S. (1991). International Quarterly of Community Health Education, 12, 89-106.

Gumperz, J. J. (1978). The conversational analysis of interethnic communication. In E. L. Ross (Ed.), Interethnic communication. Southern 
Anthropological Society Proceedings, No. 12. Athens. Vancouver: University of Georgia Press.

Gumperz, J. J. (1992). Interviewing in intercultural situations. In P. Drew \& J. Heritage (Eds.), Talk at work: interaction in institutional settings (pp. 302327). Cambridge University Press.

Gumperz, J. J., \& Tannen, D. (1979). Individual and social differences in language use. In C. Fillmore, D. Kempler \& W. S-Y. Wang (Eds.), Individual differences in language ability and language behaviour (pp. 305-325). NY: Academic Press.

Hall, E. T. (1976). Beyond culture. Doubleday, New York: Anchor Books.

Hall, J. A., Irish, J. T., Roter, D. L., Ehrlich, C. M., \& Miller, L. M. (1994). Gender in medical encounters: an analysis of physician and patient communication in a primary care setting. Health Psychology, 13, 384-392.

Hammerly, H. (1991). Fluency and accuracy: toward balance in language teaching and learning. Clevedon and Philadelphia: Multilingual matters.

Hofstede, G. (1980). Culture's consequences: international differences in work-related values. Beverly Hills, CA: Sage.

Iwao, S., \& Triandis, H. C. (1993). Validity of auto- and heterostereotypes among Japanese and American students. Journal of Cross-cultural Psychology, 24, 428-444.

Kim, Y. Y. (1978). A communication approach to acculturation processes: Korean immigrants in Chicago. International Journal of Intercultural Relations, 2, 197-224.

Kim, Y. Y. (1989). Intercultural adaptation. In M. K. Asante \& W. B. Gudykunst (Eds.), Handbook of international and intercultural communication (pp. 275-294). CA: Sage Publications.

Labov, W., \& Fanshel, D. (1977). Therapeutic discourse. NY: Academic Press.

LaFrance, M., \& Mayo, C. (1978). Moving bodies: nonverbal communication in social relationships. Inc, Belmont, CA: Wadsworth Publishing Company.

Lai, M. C., \& Yue, K. M. K. (1990). The Chinese. In N. Waxler-Morrison, J. Anderson \& E. Richardson (Eds.), Cross-cultural caring: a handbook for health professionals in western Canada (pp. 68-90). University of British Columbia Press.

Li, H. Z. (1992). Chinese Health Priority Survey in the Province of British Columbia. A report to the British Columbia Ministry of Health.

Li, H. Z. (1994). Inter- and intra-cultural information-transmission. Unpublished doctoral dissertation, University of Victoria, Victoria, BC, Canada.

Linton, R. (1936). The study of man. NY: Appleton-Century-Crofts.

Makoul, G., Arntson, P., \& Schofield, T. (1995). Health promotion in primary care: Physician-patient communication and decision making about prescription medications. Social Science Medicine, 41, 1241-1254.

Masur, F. T. (1981). Adherence to health care regimens. In C. K. Prokop \& L. A. Bradley (Eds.), Medical psychology: contributions to behavioral medicine. NY: Academic Press.

Matsumoto, D. (1992). American-Japanese cultural differences in the recognition of universal facial expressions. Journal of Cross-Cultural Psychology, $23,72-84$. 
Pierson, H. D., \& Bond, M. H. (1982). How do Chinese bilinguals respond to variations of interviewer language and ethnicity? Journal of Language and Social Psychology, 1, 123-139.

Roter, D. L. (1977). Patient participation in patient-provider interaction: The effects of patient question asking on the quality of interaction, satisfaction and compliance. Health Communication Monographs, 5, 281-315.

Roter, D. L., \& Hall, J. A. (1992). Doctors talking with patients/patients talking with doctors: Improving communication in medical visits. Westport, CT: Auburn House.

Scollon, R., \& Scollon, S. W. (1995). Intercultural communication. Blackwell Publishers Inc.

Sharf, B. F. (1990). Physician-patient communication as interpersonal rhetoric: a narrative approach. Health Communication, 2, 217-231.

Simard, L. M., \& Taylor, D. M. (1973). The potential for bicultural communication in a dyadic situation. Canadian Journal of Behaviourial Science, 5, 211225 .

Sogon, S., \& Masutani, M. (1989). Identification of emotion from body movements: a cross-cultural study of Americans and Japanese. Psychological Reports, 65, 35-46.

Stephenson, P. H. (1991). The Victoria Multicultural Research Project, a Report.

Tannen, D. (1981). Indirectness in discourse: Ethnicity as conversational style. Discourse Processes, 4, 221-238.

Taylor, D. M., \& Gardner, R. C. (1970a). Bilingual communication: a study of communicational efficiency and person perception. Canadian Journal of Behaviourial Science, 2, 67-81.

Taylor, D. M., \& Gardner, R. C. (1970b). The role of stereotypes in communication between ethnic groups in the Philippines. Social Force, 49, 271-283.

Taylor, D. M., \& Simard, L. M. (1975). Social interaction in a bilingual setting. Canadian Psychological Review, 16, 240-254.

Ting-Toomey, S. (1988). Rhetorical sensitivity style in three cultures: France, Japan, and the United States. Central States Speech Communication Journal, $38,28-36$.

Ting-Toomey, S. (1991). Intimacy expressions in three cultures: France, Japan, and the United States. International Journal of Intercultural Relations, 15, 2936.

Triandis, H. C., Leung, K., Villareal, M. J., \& Clack, F. L. (1985). Allocentric versus idiocentric tendencies: convergent and discriminant validation. Journal of Research in Personality, 19, 395-415.

Triandis, H. C., Bontempo, R., Villareal, M. J., Asia, M., \& Lucca, N. (1988) Individualism and Collectivism: cross-cultural perspectives on self-ingroup relationships. Journal of Personality and Social Psychology, 54, 323-338.

Watzlawick, P., Beavin, Bavelas J., \& Jackson, D. D. (1967). Pragmatics of human communication. New York: Norton.

Weaver, W. (1966). The mathematics of communication. In A. G. Smith (Ed.), Communication and culture (pp. 15-24). Holt Rinehart and Winston Inc.

Wiener, N. (1966). The communication of information. In A. G. Smith (Ed.), Communication and culture (pp. 15-24). Holt Rinehart and Winston Inc. 
Wood, J. B. (1989). Communicating with older adults in health care settings: cultural and ethnic considerations. Educational Gerontology, 15, 351-362.

Woon, Y. F. (1986). Some adjustment aspects of Vietnamese and SinoVietnamese families in Victoria, Canada. Journal of Comparative Family Studies, 17, 349-370.

Wyatt, N. (1991). Physician-patient relationships: what do doctors say? Health Communication, 3, 157-174. 